\title{
SOME THEOREMS ON PLANE CURVES
}

BY W. V. PARKER

In applying Abel's theorem to hyperelliptic integrals, we are interested in the intersections of certain curves with a curve $C$ of the type $y^{2}=f(x)$, where $f(x)$ is a polynomial. The functions used in the following are all polynomials of degree indicated by their subscripts. If $f_{n}(x) \equiv f_{k}(x) f_{n-k}(x)$ we may without any loss of generality assume that $n \geqq k \geqq n / 2$ and this assumption will be made throughout.

LEMma. If $C$ is the curve $y^{2}=f_{n}(x) \equiv f_{k}(x) f_{n-k}(x), c_{1}$ the curve $y=f_{k}(x)$ and $c_{2}$ the curve $y=f_{n-k}(x)$, then all the finite points of intersection of $c_{1}$ and $c_{2}$ are on $C$, and the curve $S$ whose equation is $y=\left[f_{k}(x)+f_{n-k}(x)\right] / 2$ is tangent to $C$ at each of these $k$ points.

Suppose $(\alpha, \beta)$ is any one of the $k$ points of intersection of $c_{1}$ and $c_{2}$; then $\beta=f_{k}(\alpha)$ and $\beta=f_{n-k}(\alpha)$ and therefore $\beta^{2}=f_{k}(\alpha) f_{n-k}(\alpha)=f_{n}(\alpha)$, that is $(\alpha, \beta)$ is on $C$. Obviously $S$ passes through the $k$ points of in teresection of $c_{1}$ and $c_{2}$ and hence meets $C$ in these $k$ points. Eliminating $y$ from the equations of $S$ and $C$ we get

$$
\left[\frac{f_{k}(x)+f_{n-k}(x)}{2}\right]^{2}-f_{k}(x) f_{n-k}(x) \equiv\left[\frac{f_{k}(x)-f_{n-k}(x)}{2}\right]^{2}=0
$$

as the equation giving the abscissas of the $2 k$ points of intersection of $S$ and $C$. Since the left hand side of this equation is a perfect square each abscissa is counted twice, and therefore since, in $S, y$ is a one-valued function of $x, S$ is tangent to $C$ at each of these $k$ points.

As an immediate consequence of this lemma we have the following result.

TheOREM 1. If $C$ is the curve $y^{2}=\phi_{n}(x)$, where $\phi_{n}\left(e_{i}\right)=0$, $(i=1, \cdots, n)$, and $(\alpha, \beta),(\beta \neq 0)$, is a point on $C$, and $c_{1}$ is the curve of the form $y=\phi_{k}(x)$ determined by $(\alpha, \beta)$ and any $k$ of the points $\left(e_{i}, 0\right)$, and $c_{2}$ is the curve of the form $y=\phi_{n-k}(x)$ determined by $(\alpha, \beta)$ and the remaining $n-k$ of the points $\left(e_{i}, 0\right)$, then $c_{1}$ and 
$c_{2}$ have all their $k$ points of intersection* on $C$, and the curve $S$ whose equation is $y=\left[\phi_{k}(x)+\phi_{n-k}(x)\right] / 2$ is tangent to $C$ at each of these $k$ points.

Since $\phi_{n}(x)=\phi_{k}(x) \phi_{n-k}(x)$ for $n+1$ values of $x$, we have $\phi_{n}(x) \equiv \phi_{k}(x) \phi_{n-k}(x)$ and the theorem follows from the lemma.

That all curves $S$ of the form $y=g_{k}(x)$ which are tangent to a curve $C$ of the form $y^{2}=g_{n}(x)$ at each of $k$ points can be obtained by this process, is a consequence of the following theorem.

THEOREM 2. If $\left(\alpha_{i}, \beta_{i}\right),(i=1,2, \cdots, k)$, are $k$ points on the curve $C$ whose equation is $y^{2}=g_{n}(x)$ such that there exists a curve $S$ of the form $y=g_{k}(x)$ which is tangent to $C$ at each of these $k$ points, and if the curve $c_{1}$ whose equation is $y=h_{k}(x)$ meets $C$ in the $k$ points $\left(\alpha_{i}, \beta_{i}\right)$ and the point $\left(e_{\lambda}, 0\right)$, where $e_{\lambda}$ is any zero of $g_{n}(x)$, then $h_{k}(x)$ is a factor of $g_{n}(x)$.

Since $S$ is tangent to $C$ at each of the $k$ points, the equation $g_{k}^{2}(x)-g_{n}(x)=0$ has the roots $\alpha_{1}, \alpha_{2}, \cdots, \alpha_{k}$, each counted twice, and since $c_{1}$ meets $S$ in the $k$ points $\left(\alpha_{i}, \beta_{i}\right)$, the equation $g_{k}(x)-h_{k}(x)=0$ has the roots $\alpha_{1}, \alpha_{2}, \cdots, \alpha_{k}$.

We have therefore

$$
\left[g_{k}(x)-h_{k}(x)\right]^{2} \equiv \mu\left[g_{k}^{2}(x)-g_{n}(x)\right],
$$

and hence

$$
\left[g_{k}\left(e_{\lambda}\right)-h_{k}\left(e_{\lambda}\right)\right]^{2}=\mu\left[g_{k}^{2}\left(e_{\lambda}\right)-g_{n}\left(e_{\lambda}\right)\right]
$$

but $h_{k}\left(e_{\lambda}\right)=g_{n}\left(e_{\lambda}\right)=0$, hence $\mu=1$, and we have

$$
g_{k}^{2}(x)-2 g_{k}(x) h_{k}(x)+h_{k}^{2}(x) \equiv g_{k}^{2}(x)-g_{n}(x),
$$

or

$$
g_{n}(x) \equiv h_{k}(x)\left[2 g_{k}(x)-h_{k}(x)\right] .
$$

If $c_{1}$ is the curve $y=a_{0} x^{k}+a_{1} x^{k-1}+\cdots+a_{k-1} x+a_{k}$ determined by the $k$ points $\left(\alpha_{i}, \beta_{i}\right)$ and one of the $n$ points $\left(e_{i}, 0\right)$, the coefficient $a_{0}$ may be zero and the degree of the right hand side less than $k$. For suppose we choose a particular one, say $e_{1}$, of

* Only finite points of intersection are considered here. In certain special cases when $n$ is even and $k=\frac{1}{2} n, c_{1}$ and $c_{2}$ may coincide or they may have less than $k$ finite points of intersection. The lemma and Theorem 1 are still true for these cases when finite points of intersection are considered. 
the zeros of $g_{n}(x)$ and find that the expression on the right is of degree $k$; then it will have as zeros $k$ of the zeros of $g_{n}(x)$, say $e_{1}, e_{2}, \cdots, e_{k}$. Then the curve $y=b_{0} x^{k}+b_{1} x^{k-1}+\cdots+b_{k-1} x+b_{k}$ determined by the $k$ points $\left(\alpha_{i}, \beta_{i}\right)$ and one of the remaining points $\left(e_{i}, 0\right)$, say $\left(e_{k+1}, 0\right)$, will have its right hand side of degree $n-k$ at most. For suppose the right hand side of degree $m>n-k$; then it will have as zeros $m$ of the zeros of $g_{n}(x)$ and and hence at least one of the $e_{1}, e_{2}, \cdots, e_{k}$ and therefore $a_{0} x^{k}+a_{1} x^{k-1}+\cdots+a_{k}=b_{0} x^{k}+b_{1} x^{k-1}+\cdots+b_{k}$ for at least $k+1$ values. But since $b_{0} x^{k}+b_{1} x^{k-1}+\cdots+b_{k}$ has at least one zero which is not a zero of $a_{0} x^{k}+a_{1} x^{k-1}+\cdots+a_{k}$ this is impossible. It follows as a consequence of Theorem 1 that the degree of the right hand side is either $k$ or $n-k$ depending on which zero of $g_{n}(x)$ is chosen for determining the curve $c_{1}$.

If in the above the degree of $h_{k}(x)$ is $k$, the degree of $2 g_{k}(x)$ $-h_{k}(x)$ will be $n-k$; if we denote the latter by $h_{n-k}(x)$, we shall have $g_{k}(x) \equiv\left[h_{k}(x)+h_{n-k}(x)\right] / 2$. That is, the curve $S$ is $y=\left[h_{k}(x)+h_{n-k}(x)\right] / 2$, where the curve $y=h_{k}(x)$ is determined by some $k$ of the points $\left(e_{i}, 0\right)$ and one of the points $\left(\alpha_{i}, \beta_{i}\right)$, and the curve $y=h_{n-k}(x)$ is determined by the remaining $n-k$ of the points $\left(e_{i}, 0\right)$ and the same one of the points $\left(\alpha_{i}, \beta_{i}\right)$.

Thus far it has not been necessary to say anything about the nature of the zeros $e_{1}, e_{2}, \cdots, e_{n}$. When these zeros are distinct we have the following theorem.

THEOREM 3. The number of curves of the type $y=g_{k}(x)$ which are tangent to a curve $C$ of the type $y^{2}=g_{n}(x)$ at any fixed point $(\alpha, \beta)$ and at $k-1$ other points, is $C_{k}^{n}$ for $k>n / 2$ and $\frac{1}{2} C_{k}^{n}$ for $k=n / 2$, provided that the zeros of $g_{n}(x)$ are distinct.

For by Theorem 1 we get a curve of this type corresponding to any $k$ of the zeros of $g_{n}(x)$ and by Theorem 2 all curves of this type are obtained by this process. It must be shown, therefore, that when $k>n / 2$ the same curve cannot be obtained from two different sets of $k$ zeros of $g_{n}(x)$. Suppose $y=\phi_{k}(x)$ and $y=\psi_{k}(x)$ are both of degree $k$ and cut out the same set of $k$ points $\left(\alpha_{i}, \beta_{i}\right)$ on $C$; then $\phi_{k}(x)$ and $\psi_{k}(x)$ must have at least one zero in common and therefore $\phi_{k}(x) \equiv \psi_{k}(x)$. If $n$ is even and $k=\frac{1}{2} n$, then each set of $k$ such points is cut out by two and only two of these curves by Theorem 1 .

From Theorem 1, the ordinary construction for drawing a 
tangent to a conic at a point $P$ on it, when the axes and vertices are known, follows immediately.

The following example is a rather interesting illustration of Theorem 1. Let $C$ be the curve

$$
y^{2}=f_{6}(x) \equiv-x^{6}+14 x^{4}-49 x^{2}+36 .
$$

The zeros of $f_{6}(x)$ are $1,-1,2,-2,3,-3$. Let the curve $c_{1}: y=f_{3}(x)$ be determined by $(0,6)(1,0)(-1,0)(3,0)$ and the curve $c_{2}: y=g_{3}(x)$ be determined by $(0,6)(2,0)(-2,0)$ $(-3,0)$; then we have

$$
\begin{aligned}
& f_{3}(x) \equiv 2 x^{3}-6 x^{2}-2 x+6 \\
& g_{3}(x) \equiv-\frac{1}{2} x^{3}-\frac{3}{2} x^{2}+2 x+6 .
\end{aligned}
$$

These curves $c_{1}$ and $c_{2}$ meet on $C$ in three points whose abscissas are $0,(9+\sqrt{ } 241) / 10,(9-\sqrt{ } 241) / 10$. The curve $S$ whose equation is

$$
y=\frac{f_{3}(x)+g_{3}(x)}{2} \equiv \frac{3}{4} x^{3}-\frac{15}{4} x^{2}+6
$$

is tangent to $C$ at each of these three points.

If we take for $c_{1}$ the curve $y=g_{0}(x)$ determined by $(0,6)$, and for $c_{2}$ the curve $y=g_{6}(x)$ determined by $(0,6)(1,0)(-1,0)$ $(2,0)(-2,0)(3,0)(-3,0)$, we get

$$
g_{0}(x) \equiv 6, \quad g_{6}(x) \equiv-\frac{1}{6} x^{6}+\frac{7}{3} x^{4}-\frac{49}{6} x^{2}+6 .
$$

The curves $c_{1}$ and $c_{2}$ are each tangent to $C$ at each of the three points $(0,6)(\sqrt{ } 7,6)(-\sqrt{ } 7,6)$ and the curve $S$ whose equation is

$$
y=\frac{g_{0}(x)+g_{6}(x)}{2}=-\frac{1}{12} x^{6}+\frac{7}{6} x^{4}-\frac{49}{12} x^{2}+6
$$

meets $C$ four times at each of the three points.

The University of North Carolina 